voluntarily recruited through the North East GP Vocational Training Scheme. The data was analysed using thematic analysis.

Results The findings emphasise the importance of palliative care experience during training and the value of hospice placements. The findings demonstrated that many of the skills attained through working with palliative patients were transferrable and enhanced the overall practice of the trainees. Opportunities to develop complex communication skills were particularly valued by the trainees. The interviews highlighted that there were sometimes challenges in accessing palliative care experience in community placements. The interviews were conducted during the Covid-19 pandemic and this had resulted in trainees having opportunities to gain additional experience in advance care planning.

Conclusions The study acknowledged that learning does not stop at CCT however the trainees generally felt that during training they received good preparation for independent practice in palliative care. The study supported the importance of good palliative care teaching and experience during training for managing both palliative care patients and providing transferrable holistic skills helpful in all aspects of clinical practice. Further work could be done to explore the variability of trainees' exposure to palliative care patients in the community and how trainers and practices could be supported in providing this experience.

\section{3 'MAKING HUMAN CONNECTIONS' AN EDUCATIONAL VIDEO TO ENGAGE AND PROMOTE A CULTURE OF INCLUSIVITY FOR CLINICAL AND NON-CLINICAL STAFF PROVIDING END OF LIFE CARE IN AN ACUTE LONDON NHS TRUST}

Kate Powell, Sarah MacDonald, Jessica Wood. Chelsea and Westminster Hospital NHS Foundation Trust

\subsection{6/spcare-2021-PCC.61}

Background Health professionals and ancillary staff have a unique opportunity to make a positive difference to the end of life (EOL) experiences of their patients and those who are important to them. In order to do so, they need to be equipped with a range of skills, knowledge, attitudes and behaviours. The End of Life Care Core Skills Education and Training Framework sets out the skills and knowledge to achieve this. This framework was used to benchmark the current provision of EOL training and education at a Londonbased NHS Trust and to develop a novel approach to meeting identified gaps.

Methods A task and finish group consisting of members of the Trust's specialist palliative care team and the EOL care clinical leads reviewed the education mapping exercise and identified key areas of focus for the Trust's EOL education and training programme. These were triangulated with other national EOL frameworks, audits and quality standards.

Results The key education themes identified were: 1) personalisation of end of life care (finding out 'what matters most' to each patient); 2) every staff member has a role in EOL care. Non-clinical staff involved with patients at the end of life were identified as one of the key target groups in the EOL education gap analysis. A short video was agreed as the most innovative educational resource with a focus on the core themes for a non-clinical target audience. In collaboration with an external production team, the narratives of ten Trust employees from a diverse range of clinical and non-clinical roles were recorded.

Conclusion This quality improvement initiative sought to innovatively address a gap in EOL education through the development of an educational video with contributions from a diverse range of clinical and non-clinical staff. The next steps are to implement and evaluate the impact of this project.

\section{PALLIATIVE SIMULATION FOR INTERNAL MEDICINE TRAINEES (PALL-SIM-IM): A NATIONALLY ADOPTED EDUCATION INITIATIVE IN RESPONSE TO SHAPE OF TRAINING}

Kate Howorth, Kerry Waterfield, Felicity Dewhurst, Emily Kavanagh, Jo Brown, Hannah Billett, Elizabeth Fleming, Rachel Kiltie, Elizabeth Woods, Grace Rowley, Max Charles, Amy Huggin, Craig Gouldthorpe, Lucy Robinson, Charlotte Bryan, Lauri Simkiss. North East Palliative Registrars Research Alliance (NEPRRA)

\subsection{6/spcare-2021-PCC.62}

Background Shape of Training represents a significant change in postgraduate-medical-education. Internal Medicine trainees (IMT) must prove competency in 8 Clinical Capabilities in Practice (CiPs). CiP 8 Managing End-of-Life and Applying Palliative Care Skills represents an opportunity to improve palliative medicine education for generalists, however, the provision of the required education and training is also a significant challenge requiring consideration and innovation. Simulation has been recognised by the Joint Royal College of Physicians Training Board (JRCPTB) as a holistic teaching and assessment method.

Aim To produce a simulation training package for IMTs covering all components/descriptors of CiP 8 which can be effectively delivered by a combination of Palliative Medicine Trainees (PMTs), consultants, simulation technicians and teaching fellows. To enable IMTs to learn/practice required skills whilst PMTs address teaching and management curriculum competencies.

Methods The North East Palliative Registrars Research Alliance (NEPRRA) (a unique initiative ensuring all North East PMTs are involved in teaching/research projects) received simulation training and designed Palliative Simulation for Internal Medicine Trainees (PALL-SIM-IM) a package comprising scenarios mapped to IMT curriculum descriptors. Modification occurred based on feedback and new scenarios incorporated requirements unique to the COVID-19 pandemic.

Results PALL-SIM-IM has been effectively piloted in 6 sessions throughout the North-East, training 25 IMTs/equivalent. It has received excellent qualitative feedback. Quantitative data demonstrates significant improvement in competence in all curriculum descriptors following training. PALL-SIM-IM has been presented (on request) to IM Specialist Trainee Committees, Health Education England and the JRCPTB. PALL-SIM-IM has been requested by, distributed to and is to be adopted by 7 Health Education England training regions, Scotland and Northern Ireland.

Conclusions This educational initiative has successfully produced a nationally adopted simulation-based training package for IMTs to aid competency development in CiP 8, reducing duplication of work whilst allowing reciprocal development of PMTs' leadership and teaching skills. 